ORIGINAL ARTICLE

\title{
The Frequency of Natal and Neonatal Teeth in a Tertiary-care Hospital, Southern Punjab
}

\author{
RABIA ZAFAR ${ }^{1}$, JAFFAR HUSSAIN BUKHARI ${ }^{2}$, MOHAMMAD RAUF AHMED ${ }^{3}$, SANA CHAUDHRY ${ }^{4}$ \\ ${ }^{1}$ Associate Professor of Operative Dentistry, Dental Section, Multan Medical and Dental College, Multan \\ ${ }^{2}$ Assistant Professor of Dental Materials, Nisthar Institute of Dentistry, Multan \\ ${ }^{3}$ Assistant Professor of Operative Dentistry, Nishtar Institute of Dentistry, Multan \\ ${ }^{4}$ Associate Professor Oral Biology Department Avicenna Dental College \\ Correspondence to: Dr. Mohammad Rauf Ahmed, Email ID: drmohammadrauf@gmail.com, Cell No: 0300-2129676
}

\begin{abstract}
Objective: To find out the frequency of natal and neonatal teeth in local population of Southern Punjab.

Material and methods: This was an observational prospective study conducted in dental outdoor department of Ibn-e-Sina Hospital, Multan from July 2019 to July 2021 after taking approval of the ethical committee. A total of 3800 patients were included. An informed consent was taken and neonates upto the age of six years were included in the study and data was collected about age, sex, religion, mother condition before and after delivery, family history and infection during pregnancy.

Results: A total of 3800 children were examined during the study period and out of which 7 children have natal and neonatal teeth and natal teeth are more common than prenatal teeth (5:2) with a ratio of $2.5: 1$. The prevalence of natal and neonatal teeth during this period was 1:543. Data showed a female predominance with male to female ratio of $1: 2.5$ and $100 \%$ were mandibular incisors.

Conclusion: It can be concluded from the study that natal and neonatal teeth are benign condition of oral cavity with frequency of $0.18 \%$. These have female predilection and mandibular incisors are the most frequent type. Ventral tongue trauma is frequent problem faced by baby and these have no relation to the mode of delivery.

Keywords: Natal, neonatal, mandibular incisors, teeth.
\end{abstract}

\section{INTRODUCTION}

Tooth eruption is a normal physiological phenomenon in human life. The tooth eruption has its own time frame, when it occurs before time, this may cause problem for mother, baby and family. It was define as natal and neonatal on the basis of time of eruption. Natal are the tooth present at the time of birth and neonatal are the tooth which erupt during first one to four weeks of birth. ${ }^{1}$

The reports about natal and neonatal teeth are found in ancient history, during roman times by Titus Livis (59BC) and in 23BC by Caius Plinius Secundus described about Natal and neonatal teeth. During that period, there were superstitions about natal and neonatal teeth. In some countries it was a symbol of success and fame, and in others countries it was sign of misfortune. In others it was extracted to get rid of evil spirits. ${ }^{2}$

Aetiology of natal and neonatal teeth is not exactly known. There are many factors that are proposed to be the cause of early eruption of teeth. These include the nutritional status, trauma, endocrine or hormonal stimulation, inheritance of autosomal dominant trait, environmental factors, and superficial position of tooth germ osteoblastic activity in the area of tooth germ. ${ }^{3}$ These are associated with different syndromes also. Studies have shown that $2 \%$ and $10 \%$ of infants with natal and neonatal teeth have unilateral and bilateral cleft palate respectively. These are also associated with Ellis-Van Creveld, Jadassonhn-Lewandowsky, Hallerman-Streiff and Pierre Robbin ets ${ }^{2,4}$

The prevalence of the natal and neonatal teeth varies greately, ranging from 1:1000 to $1: 30000$ live births. The commonest site for the natal teeth is mandibular incisors (85\%), maxillary incisors (11\%), mandibular canine and molar (3\%) and maxillary canine and molar (1\%). Only $10 \%$ of these are supernumerary teeth, rest of all are primary teeth. Among these natal teeth are more common than neonatal teeth. It is shown by studies that natal teeth have female predominance and even some studies have shown that it is more prevalent in muslim kids. ${ }^{2,3,4,5,6}$ Natal and neonatal teeth are associated with several issues, which are faced by mother and baby. Some of these issues are discomfort during feeding, breast trauma even laceration, ulceration in mouth especially in sublingual area and risk of aspiration of tooth. ${ }^{1,2}$

So aim of the current study was to know the frequency of natal and neonatal teeth and problems associated with it. It requires special attention because of the problems associated with it. So identification and addressing problems related to this early can ease both mother and baby.

\section{MATERIAL AND METHODS}

This was an observational prospective study conducted in dental outdoor department of Ibn-e-Sina Hospital, Multan from July 2019 to July 2021 after taking approval of the ethical committee. A total of 3800 patients who presented to the hospital were examined for the presence of natal and neonatal teeth. An informed consent was taken from the parent after explaining them the procedure. The neonates up to the age of six years were included in the study and predesigned proforma was filled which included information about age, sex, religion, mother condition before and after delivery, family history and infection during pregnancy. All children were undergone clinical dental examination to see the location, number and any complication. Instruments used during examination included mouth mirror, torch, and gloves.

The variables were analysed for gender, type of tooth, family history and clinical characteristics by using Chi 
square test to see possible association and a $p$ value of less than 0.05 was set as a level of significance.

\section{RESULTS}

A total of 3800 children were examined during the study period and out of which 7 children have natal and neonatal teeth. In this study it was found that Natal teeth are more common than prenatal teeth $(5: 2)$ with a ratio of $2.5: 1$. The prevalence of natal and neonatal teeth during this period was 1:543 as shown in figure 1. Data showed a female predominance with male to female ratio of 1:2.5 as shown in figure 2. The difference was not statistically significant with $p$ value of 0.063 . In this study $100 \%$ were mandibular incisors.

Relationship with mode of delivery was also noted, among positive cases 4 were born by $\mathrm{C}$-section and 3 with normal delivery. When evaluated for relationship, there was no significant difference between these with $p$ value of 0.08 . Majority babies were with natal and neonatal teeth first born baby and only one baby was second born baby.

Data showed that all 7 cases had ventral tongue trauma and it was followed by inflammation of gingival and difficulty in feeding, given in figure 3.

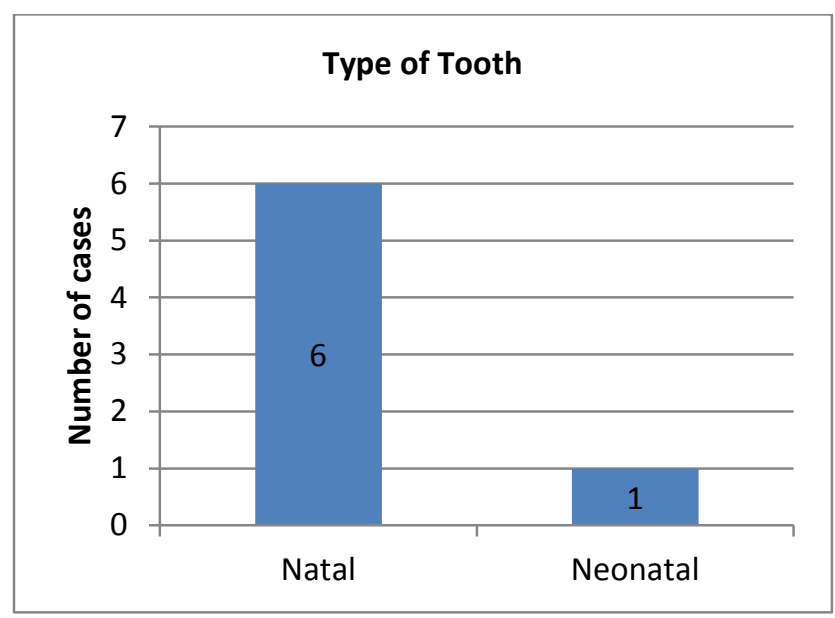

Figure 1. Type of different teeth

\section{Gender distribution}

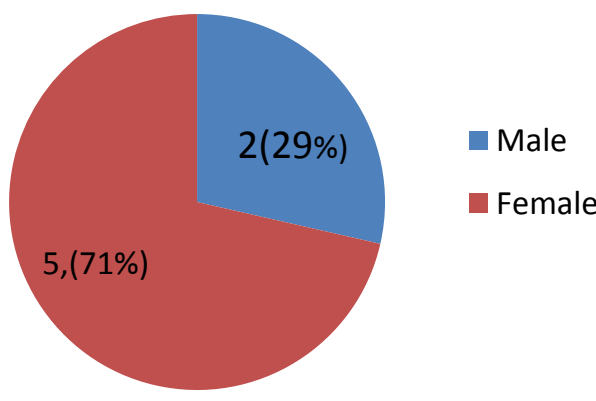

Figure 2. Gender distribution of babies with natal and neonatal teeth

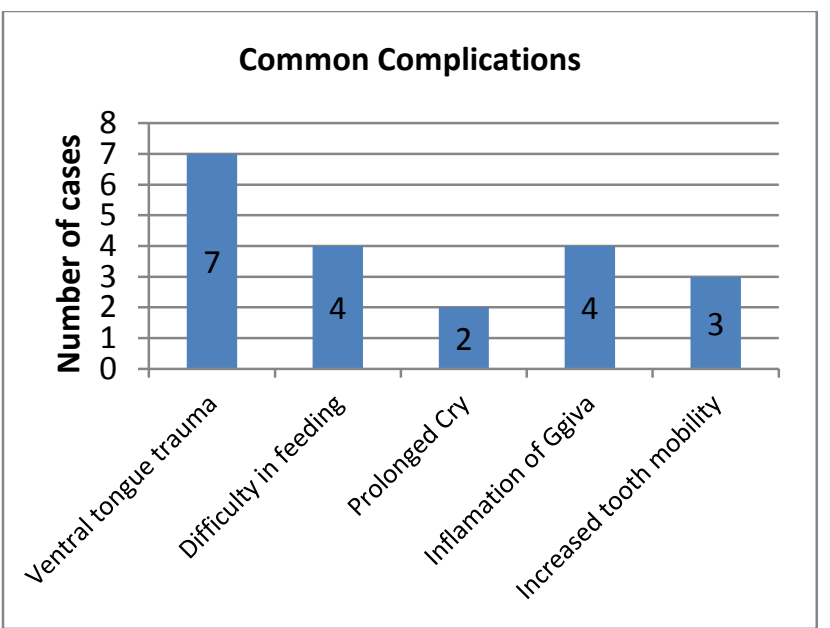

Figure 3. Common problems associated with natal and neonatal teeth

\section{DISCUSSION}

For the improvement of health and physical wellbeing of children, oral health matters a lot. Even after the so much emphasis on oral health, global evidence report showed that nearly $90 \%$ school going children have bad oral health. ${ }^{7}$ So present dentistry give much importance to give full care to children especially during first year of life. For this, it is important to know the issues presenting during this period. ${ }^{8}$

One such problem though rare, faced by infants in natal and neonatal teeth. Normally teeth erupt after six months of life, but presence of these teeth before time will produce anxiety in mother and family. Many factors are proposed by previous studies, like trauma, infections, heredity, superficial position of germ plate and environmental. In environmental polychlorinated biphenols, polychlorinated dibenzo-p-dioxin and dibenzo-furans are suggested to be the cause of natal and neonatal teeth. These are most frequent pollutants in environment. ${ }^{1,9}$

Literature has shown that natal teeth are more prevalent than neonatal teeth. So is the finding of the present research. One study by Mirza et al at Karachi has also shown that natal teeth are the common one. ${ }^{1}$ Studies from India have similar results. ${ }^{6.10,11}$ The frequency of natal and neonatal teeth in present study was $0.18 \%$, one study from India have frequency of $0.16 \%$ which is similar to our study. ${ }^{10}$

Natal and neonatal teeth have predilection for females as proposed by previous studies. The current study confirmed this. Mirza et al also have same findings. ${ }^{1}$ Gunasekaran et al in India also described in their study that it is more common in females. ${ }^{10}$ Due to the low frequency of the natal and neonatal teeth, statistical significance after comparison between groups cannot be found.

In the current study mode of delivery was also inquired and was not found to be significantly related to the natal and neonatal teeth. An Indian study also describe same findings. ${ }^{5}$

In literature, it was described that most frequent position of the natal and neonatal teeth are mandibular incisors followed by maxillary incisors. ${ }^{1,10,12}$ In the current study $100 \%$ cases have mandibular incisors. Natal and 
neonatal teeth are proposed to be associated with many syndromes like Rubinstein-taybi, pachyonchia conginta and Hallermann-streff etc. It is also said that natal and neonatal teeth are seen at increased rate in children with cleft lip. ${ }^{13,14,15,16}$ In this study none of children have any syndrome associated with these.

Different issues related to natal and neonatal teeth are faced by baby and mother. Some of these are tongue ulceration, feeding issues, breast trauma, prolonged cry, risk of aspiration of teeth. 1,4 Current study found that $100 \%$ children have ventral tongue ulceration followed by feeding issues and prolonged cry. So the findings are similar to the previous studies.

\section{CONCLUSION}

It can be concluded from the study that natal and neonatal teeth are benign condition of oral cavity with frequency of $0.18 \%$. These have female predilection and mandibular incisors are the most frequent type. Ventral tongue trauma is frequent problem faced by baby and these have no relation to the mode of delivery.

Recommendations: Further research needed to establish the aetiology of the condition and association with risk factors. Population based research needed to know the incidence in general population.

Limitations: It was a single centre study and small sample size, so results cannot be generalized to the general population.

\section{REFERENCES}

1. Mirza D, Taqvi GH, Ikram K, Ahmed S, Hakeem S. Natal and neonatal teeth-an uncommon oral finding: a cross sectional study in pakistani subjects. Pakistan Oral \& Dental Journal. 2015;35(1).39-43.

2. Leung AK, Robson WL. Natal teeth: a review. Journal of the national medical association. 2006 ;98(2):226-228.

3. Wang $\mathrm{CH}$, Lin YT, Lin YT. A survey of natal and neonatal teeth in newborn infants. Journal of the Formosan Medical Association. 2017;116(3):193-6.
4. Bulut G, Bulut H, Ortac R. A comprehensive survey of natal and neonatal teeth in newborns. Nigerian journal of clinical practice. 2019;22(11):1489-1494.

5. Yen VA, Kuppuswami N. Incidence of Natal Teeth in Newborns in Government Medical College and Hospital, Chengalpattu: A Pilot Study. Journal of clinical and diagnostic research: JCDR. 2017;11(4):ZC86-ZC88.

6. Patil A, Shigli AL, Mehta SD, Zaparde NN. Natal Tooth-An Overview and A Case Report. Dentistry. 2017;7(405):21611122.

7. Batra M, Pirvani M, Shah AF, Gupta M. Oral Health Disparities among 12-15 Years Children of India and Pakistan-A Cross Border Comparison. JPDA. 2014;23(04):170-174.

8. Cunha RF, Boer FA, Torriani DD, Frossard WT. Natal and neonatal teeth: review of the literature. Pediatric dentistry. $2001 ; 23(2): 158-62$.

9. Mhaske S, Yuwanati MB, Mhaske A, Ragavendra R, Kamath K, Saawarn S. Natal and neonatal teeth: an overview of the literature. International Scholarly Research Notices. 2013;2013.

10. Gunasekaran G, Mani G, Rengalakshmi S. Prevalence of natal or neonatal teeth in paediatric dental patients in chennai-a retrospective study. PalArch's Journal of Archaeology of Egypt/Egyptology. 2020;17(7):217-26.

11. Agrawal NK, Khatry SK, LeClerq SC, Katz J, Mullany LC. Incidence of Natal Teeth in Sarlahi District of Nepal. Journal of Nepal Health Research Council. 2019;17(1):100-2.

12. Al-Buainain FS, Adil A, Mustafa O. Natal and Neonatal Teeth in Bahrain: A Review of 19 Cases.J Bahrain med soc.2021;33(1):31-37.

13. Malki GA, Al-Badawi EA, Dahlan MA. Natal teeth: a case report and reappraisal. Case reports in dentistry. 2015 Feb 4;2015.

14. Yilmaz RB, Cakan DG, Mesgarzadeh N. Prevalence and management of natal/neonatal teeth in cleft lip and palate patients. Eur J Dent 2016;10:54-8.

15. Yilmaz R, Cakan D, Mesgarzadeh N. Prevalence and management of natal/neonatal teeth in cleft lip and palate patients. European Journal of Dentistry. 2016; 10: 054-058.

16. Triches $\mathrm{T}$, Mondardo B, Triches R, et al . Natal and neonatal teeth: two clinical cases report. Rev Gaúcha Odontol. 2018; 66: 101-105. 